\title{
Malária no Piauí: espacialização dos casos no período de 2008 a 2013
}

\section{Malaria in Piauí: spatialization cases in the period 2008 to 2013}

DOI: $10.46919 / \operatorname{archv} 1 n 5-006$

Recebimento dos originais: 10/07/2020

Aceitação para publicação: 30/08/2020

\section{Antonio Carlos dos Santos}

Doutorando em Desenvolvimento e Meio Ambiente rede PRODEMA/UFPI

Instituição: Universidade Federal do Piauí, Teresina - PI

Endereço: Av. Universitária, 1310 (TROPEN) 64.049-550 Teresina - Piauí - Brasil

E-mail: baulive@ hotmail.com

\section{Gustavo Souza Valladares}

Doutor em Agronomia pela Universidade Federal Rural do Rio de Janeiro Universidade Federal do Piauí, Teresina - PI

Endereço: Campus Ministro Petrônio Portela - UFPI - Ininga, Teresina -PI, Brasil.

E-mail: valladares@ufpi.edu.br

\section{Izabella Cabral Hassum}

Doutora em Ciência Animal pela Universidade Federal Rural do Rio de Janeiro

EMBRAPA - Empresa Brasileira de Pesquisa Agropecuária

Avenida Duque de Caxias, 5650 - Buenos Aires, Teresina - PI, Brasil

E-mail: Izabella.hassum@embrapa.br

\section{Mauro Fernando Barbosa Chagas}

Graduado em Saúde Publica pela Universidade Estadual do Piauí.

Secretaria Estadual de Saúde do Piauí - SESAPI

Endereço: Av. Pedro Freitas, s/no, Bloco A - Centro Administrativo, bairro São Pedro, Teresina - PI

E-mail: mauropiaui@yahoo.com.br

\section{Mônica da Silva Pinto Cronemberger}

Bacharelado em Enfermagem pelo Centro Universitário Uninovafapi

Especialista em Epidemiologia pela Fundação Osvaldo Cruz - Fiocruz - RJ

Secretaria Estadual de Saúde do Piauí - SESAPI

Endereço: Av. Pedro Freitas, s/nº, Bloco A - Centro Administrativo, bairro São Pedro, Teresina - PI

E-mail: mpberger@bol.com.br

\section{RESUMO}

A malária é uma doença infecciosa, aguda, febril transmitida pela picada da fêmea de mosquito do gênero Anopheles. No Brasil, a região amazônica é endêmica para transmissão da malária onde ocorrem 99,6\% dos casos. O estado do Piauí notificou no período de 2008 a 2013, 279 (duzentos e setenta e nove) casos do agravo, sendo 112 (cento e doze) autóctones todos em sua maioria residentes na zona rural e 169 (cento e sessenta e nove) importados. Este estudo tem como objetivo realizar análise da espacialização dos casos de malária no Piauí do ano de 2008 a 2013. Os resultados obtidos através dos dados do SINAN e por meio do uso do SIG foram seis mapas espacialização da doença no estado com o número de caso absolutos, ano a ano, durante o período estudado. Na discussão identificou-se que o padrão de 
distribuição espacial dos surtos de casos de malária está concentrado na porção noroeste do Piauí na fronteira com o Maranhão. Após as análises, conclui-se que todos os 58 municípios que registraram malária no Piauí na série temporal do estudo, estão localizados e aglomerados em área vulnerável e receptiva para a transmissão da doença.

Palavras-chave: 1.Anopheles, 2.Espacialização, 3.Malária, 4.SIG.

\begin{abstract}
Malaria is an infectious disease, acute, febrile transmitted by the bite of the female mosquito of the genus Anopheles. In Brazil, the Amazon region is endemic for malaria transmission where there are $99.6 \%$ of cases. The state of Piaui notified from 2008 to 2013, 279 (two hundred and seventy nine) of grievance cases and 112 (one hundred and twelve) all mostly indigenous residents in the countryside and 169 (one hundred and sixty-nine) imported. This study aims to conduct analysis of the spatial distribution of malaria cases in Piauí of 2008 to 2013. The results obtained through SINAN data and through the use of GIS were six maps spatial distribution of the disease in the state with the number of case absolute, year by year during the study period. In the discussion it was found that the pattern of spatial distribution of malaria cases outbreaks are concentrated in the northwestern portion of Piaui on the border with Maranhão. After analysis, it is concluded that all 58 municipalities that malaria recorded in Piauí in the time of the study series, and clusters are located in vulnerable and receptive area for disease transmission.
\end{abstract}

Keywords: 1.Anopheles, 2.Espacialização, 3.Malária, 4.SIG.

\title{
1 INTRODUÇÃO
}

A malária é uma doença infecciosa, aguda, transmissível, causada por protozoários do gênero Plasmodium (SANTOS et al., 2014; SANTOS, 2015). A transmissão dos parasitos se dá através da picada da fêmea do mosquito do gênero Anopheles popularmente conhecido como mosquito prego.

Segundo Brasil (2002) a malária é uma enfermidade que ocorre em cerca de 90 países, localizados principalmente na faixa entre os trópicos de Câncer e Capricórnio. A Organização Mundial da Saúde (OMS) considera a malária um grande problema de Saúde Pública nos países em desenvolvimento. Estima-se que 300 a 500 milhões de pessoas sejam infectadas a cada ano e que nos países da África estão concentradas $90 \%$ dessas pessoas. Mais de dois bilhões de pessoas em todo mundo vivem em áreas onde há risco de infecção e 270 milhões estão parasitados.

No Brasil, esta doença é endêmica na região amazônica responsável por 99,6\% dos casos (COSTA et al., 2014). Os outros casos acontecem na área extra Amazônia cujos registros são esporádicos e isolados. De acordo com Santos et al., (2013), no mundo cerca de 380 espécies de anofelinos, são descritos, no entanto, apenas 60 são capazes de transmitir a doença. Cinco espécies no Brasil têm maior envolvimento na transmissão da malária humana, as quais são: A. (Nyssorhynchus) darlingi, A.(Nys.) aquasalis, A.(Nys.) albitarsis, A.(Kerteszia) cruzii, A.(Kerteszia) bellator. 
O estado do Piauí, segundo Chagas et al., (2013), é considerado como uma área livre da transmissão da doença desde meados da década de 1985, apresentando, porém, surtos ocasionais de malária desde o ano 2000.

O Piauí notificou no período de 2008 a 2013, 279 (duzentos e setenta e nove) casos de malária, sendo 112 (cento e doze) autóctones todos em sua maioria residentes na zona rural e 169 (cento e sessenta e nove) importados. A problemática da ampla distribuição de casos de malária no estado do Piauí deixa clara a necessidade de outras ciências atuarem em conjunto com as vigilâncias em saúde para a realização de estudos que direcionem métodos preventivos e técnicas de controle no combate à doença.

Diante do exposto elaborou-se a seguinte hipótese para a realização do estudo: A espacialização da malária no estado do Piauí do ano de 2008 a 2013 está concentrada e aglomerada na região norte do estado. Assim o presente estudo teve como objetivo realizar análise da espacialização dos casos de malária no Piauí durante o período de 2008 a 2013, utilizando o sistema de informação geográfica para mapeamento dos casos da doença, os quais permitem investigar o comportamento da incidência do agravo no período estudado e identificar o padrão de distribuição espacial.

\section{MATERIAIS E MÉTODOS}

\subsection{TIPO DE ESTUDO}

Neste trabalho além do estudo geográfico ambiental foi utilizado também o epidemiológico do tipo ecológico e ambiental. O geográfico ambiental que possibilita o entendimento da dinâmica da organização espacial (CAVALCANTI, 2006). E o último o ecológico, pois se refere à área geográfica estudada, com variáveis ambientais, habitacionais e indicadores de saúde (FILHO et al.,1990). Para as análises dos casos de malária trabalhou-se os dados em uma escala de padrão espacial em todo território do estado do Piauí.

\section{2 ÁREA DE ESTUDO}

A área de estudo, o estado Piauí (PI), está localizado na região Nordeste do Brasil (Figura 1), geomorfologicamente é composto por planaltos e planícies. O PI possui uma área de 251.273,186km² e limita-se ao norte pelo Oceano Atlântico paralelo de $2^{\circ} 44^{\prime} \mathrm{S}$, ao sul pelo paralelo $10^{\circ} 52^{\prime}$ 'S, a leste pelo divisor de águas dos riachos Riachão e Conceição ( $\left.40^{\circ} 22^{\prime} \mathrm{W}\right)$, e a oeste pelo rio Parnaíba (45⒌'W) entre os afluentes da margem direita, rio da Pedra Furada e rio Medonho (RODRIGUES, 2012). 
Figura 1: Mapa de localização da área de estudo

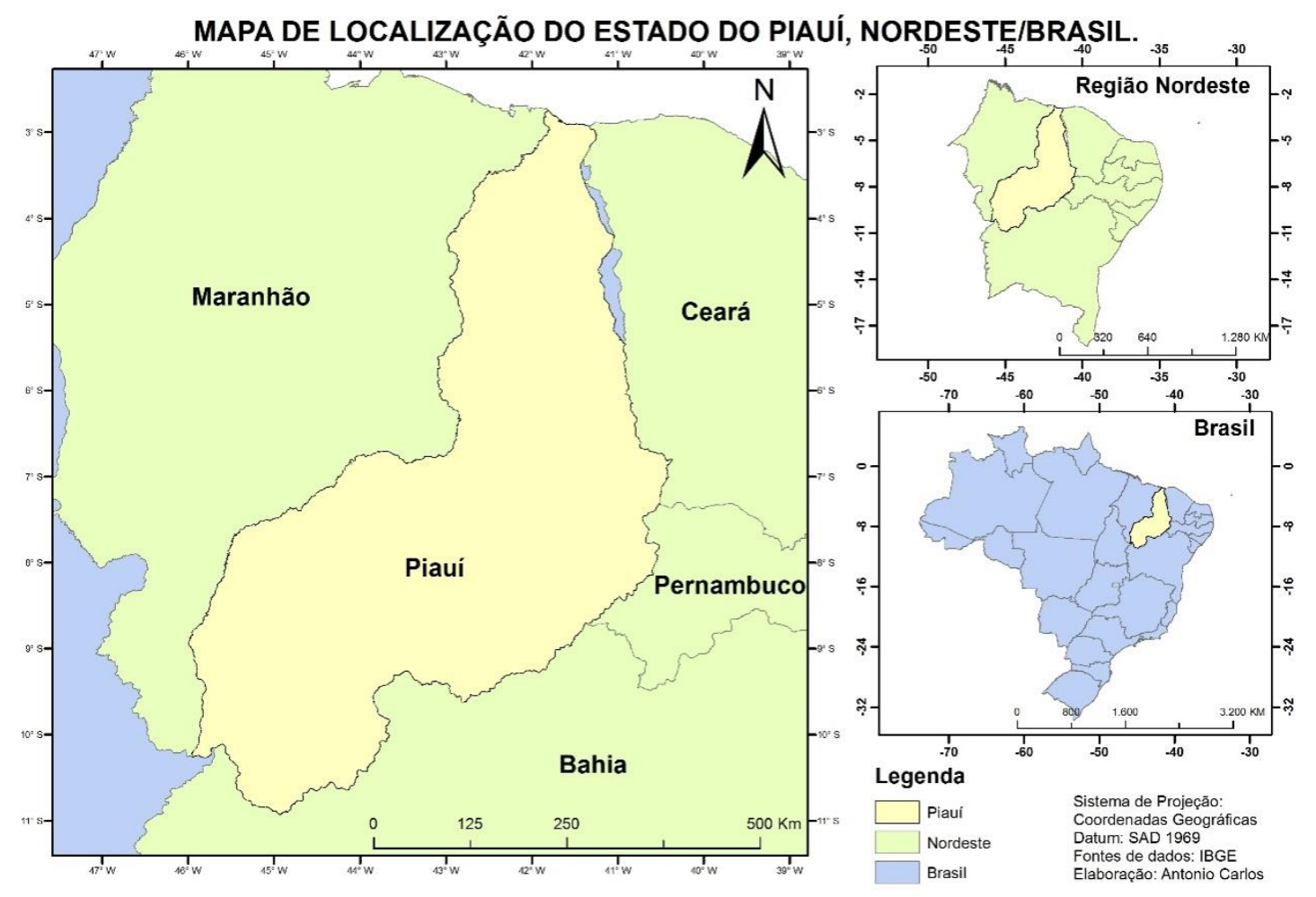

[Fonte: Mapa índice do Brasil - IBGE]

O Piauí, atualmente conta uma população geral de 3.119.015 habitantes, sendo um total de 1.528.796 de homens e 1.590.219 o total de mulheres segundo o Censo Demográfico (IBGE, 2012). Está dividido em 224 municípios, é o terceiro maior estado nordestino e o décimo primeiro estado brasileiro em extensão territorial. Pertence ao segundo fuso horário brasileiro.

Neste trabalho se optou em realizar uma análise com recorte espacial de 58 municípios dos 224 existentes na atual divisão política administrativa do estado devido os mesmos possuírem registro de casos de malária durante o período de 2008 a 2013 (série de estudo). O quadrilátero do Piauí (Figura 1) é uma região que apresenta uma variedade de ecossistemas, por estar inserido em uma zona de transição entre os biomas amazônico, cerrados e caatinga (RODRIGUES, 2012). Segundo EMPERAIRE (1985) citado por Rodrigues (2012) a classificação da vegetação está distribuída em: cerrado, caatinga, palmeiras, vegetação litorânea e áreas de transição.

\subsection{PLANO AMOSTRAL}

A população do estudo foi constituída pela totalidade dos casos notificados e confirmados de malária informados à Secretaria de Estado de Saúde do Piauí (SESAPI) no período de 2008 a 2013 no Sistema de Informação de Agravos de Notificação (SINAN). O SINAN é o sistema de informação 
recomendado pelo Ministério da Saúde (MS) para registro da malária na região extra Amazônia. As informações foram geradas por um instrumento de coleta de dados, denominado de Ficha de Investigação Epidemiológica (FIE) de malária.

\subsection{COLETA E TRATAMENTO DE DADOS}

Em relação à coleta dos dados epidemiológicos da malária foram utilizadas informações estatísticas da SESAPI e os demográficos do Instituto Brasileiro Geografia e Estatística - IBGE. Os dados do SINAN são gerados pelas Secretarias Municipais de Saúde (SMS), seguindo para as Secretarias Estaduais de Saúde (SES) e posteriormente, enviadas para o nível nacional, para Gerência Técnica do Programa Nacional de Controle da Malária, por meio eletrônico. Estes dados foram analisados quanto às possíveis incoerências e organizados em tabelas.

Os mapas temáticos de espacialização do número total dos casos de malária no Piauí foram gerados na base municipal vetorial em SIG, que resultaram em 06 mapas da doença equivalentes ao número de anos do período de estudo (2008 a 2013).

\section{RESULTADOS E DISCUSSÃO}

A malária nos anos de 1970 e até 1986 apresentava-se como um grande problema de saúde pública no Piauí, tendo sido verificado desde então uma sensível diminuição nos casos, sejam eles provenientes de outras regiões (casos importados) ou originários do próprio Estado (casos autóctones).

Fazendo uma avaliação da série histórica de 2008 a 2013 de casos de malária (Tabela 01), observou-se que a cada ano do período foram notificados casos de malária autóctone. Identificou-se também que somente nos anos de 2008 e 2009 da série estudada a ocorrência de malária não ultrapassou 25 casos. Do total de 279 casos de malária registrados na série, 112 foram autóctones o que representa cerca de $40,1 \%$ dos casos do período estudado. Situação que comprova que o estado do Piauí é vulnerável e receptivo ao aparecimento de surtos de malária em uma vasta área geográfica do seu território. Desde 1986 o Piauí é considerado como área de vigilância para transmissão da malária; devido ao fato, pode-se considerar o risco iminente do restabelecimento da malária como endemia no estado se não houver um maior comprometimento em controlar uma possível evolução dos autóctones.

Tabela 01 - Série Histórica da Malária - Piauí, período: 2008 - 2013

\begin{tabular}{cccc}
\multicolumn{4}{c}{$\mathbf{N}^{\mathbf{0}}$ DE CASOS DE MALÁRIA } \\
\cline { 2 - 3 } ANO & AUTÓCTONES & IMPORTADOS & TOTAL \\
\hline 2008 & 8 & 17 & 25 \\
2009 & 13 & 8 & 21 \\
2010 & 45 & 28 & 73
\end{tabular}




\begin{tabular}{ccccc}
2011 & 15 & 50 & 65 \\
2012 & 13 & 34 & 47 \\
2013 & 18 & 30 & 48 \\
\hline Total & 112 & 167 & 279 \\
\hline
\end{tabular}

[Fonte: SINAN NET/SESAPI]

Em 2008, início da série de estudo, 13 municípios do estado do Piauí de acordo com a Figura 2 notificaram 25 casos de malária, sendo oito autóctones e 17 importados. O município de Barras notificou o maior número de casos deste ano no total de cinco sendo todos importados. Em seguida o município de Teresina notificou quatro casos, sendo dois autóctones e dois importados. Depois de Teresina mais cinco municípios notificaram casos autóctones, são eles Porto com 02 casos e Luzilândia, Buriti dos Lopes, Matias Olimpio e Nossa Senhora dos Remédios com um caso cada. Observa-se pelo mapa que o padrão de distribuição espacial da malária este ano aglomerou-se na região Norte do estado excetuando-se o município de Teresina que sua localização fica mais ao centro. Percebe-se que a área geográfica de atuação da malária (Figura 2) limita-se da região central ao norte do estado.

No ano de 2009 segundo o mapa da Figura 3 foram notificados 21 casos de malária em 11 municípios do estado do Piauí, sendo 13 casos autóctones e oito importados. Caraúbas do Piauí e Luzilândia ambos localizados na região Norte do estado notificaram o maior número de casos de malária quatro casos cada um, sendo todos autóctones. Os municípios de Canxingó e Madeiro registraram dois casos de malária autóctones cada um e de Murici dos Portelas um caso autóctone. Totalizando cinco municípios com registro de casos autóctones em 2009. O padrão espacial da ocorrência de malária permanece parecido com o de 2008 com exceção do município de Regeneração que fica na região central do estado (Figura 3). 
Figura 2 - Mapa da distribuição espacial dos casos de malária no Piauí no ano de 2008 por município.

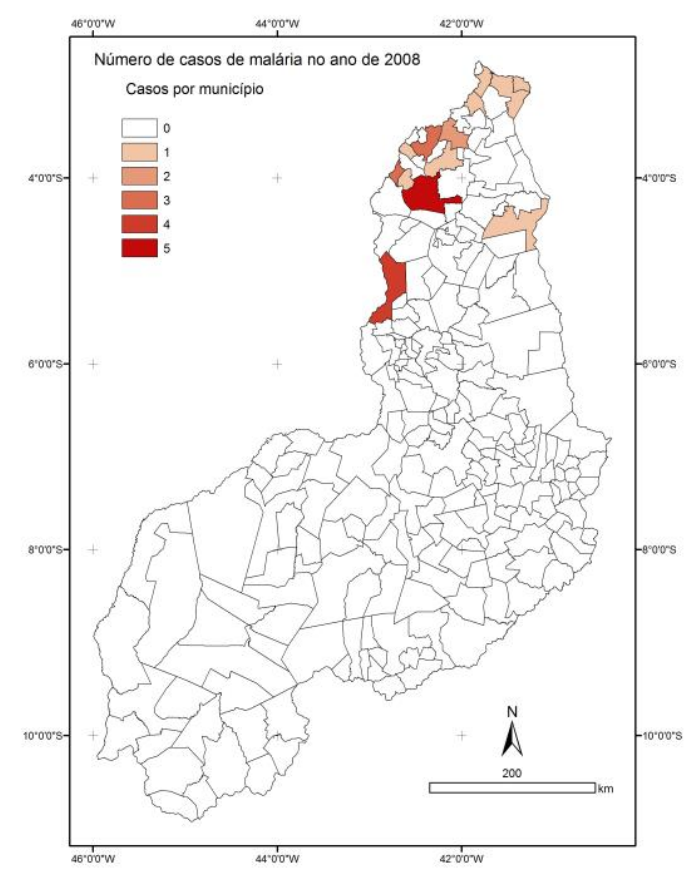

Fonte: SINAN/SESAPI, adaptado por Antonio Carlos dos Santos, 2014.
Figura 3 - Mapa da distribuição espacial dos casos de malária no Piauí no ano de 2009 por município.

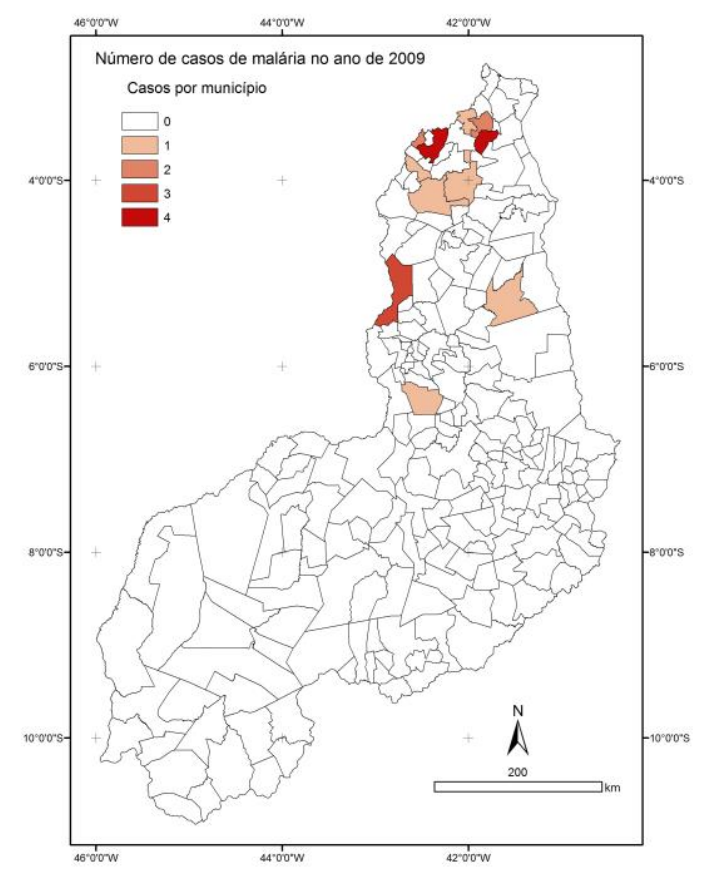

Fonte: SINAN/SESAPI, adaptado por Antonio Carlos dos Santos, 2014.

Em 2010 o padrão de distribuição dos casos de malária no estado começa a dispersar atingindo de Norte a Sul o Piauí (Figura 4). Foram notificados 73 casos de malária distribuídos em 19 municípios, sendo 45 casos autóctones e 28 importados. Neste ano foram registrados dois surtos de malária, sendo um no município de Buriti dos Lopes com 26 casos e outro em Luzilândia com 11 casos. Fora esses dois municípios outros quatro tiveram registros de casos autóctones, sendo eles: Teresina, Batalha, Matias Olímpio e Picos cada um com o registro de um caso.

Observando a abrangência geográfica da ocorrência de malária no Piauí (Figura 5) verifica-se em 2011 um maior número de municípios atingidos pela enfermidade em relação aos anos anteriores e que o padrão espacial dos casos de malária está concentrado a oeste do estado margeando o rio Parnaíba e também ao Leste do estado. Foram notificados 65 casos de malária em 2011, sendo 15 casos autóctones e 50 casos importados. O maior número de casos registrados neste ano foi em Uruçuí com 18 casos, sendo 12 autóctones e seis importados. Os municípios de Luzilândia, Buriti dos Lopes e Vera Mendes registraram um caso de malária autóctone cada um. 
Figura 4 - Mapa de distribuição espacial dos casos de malária no Piauí no ano de 2010 por município

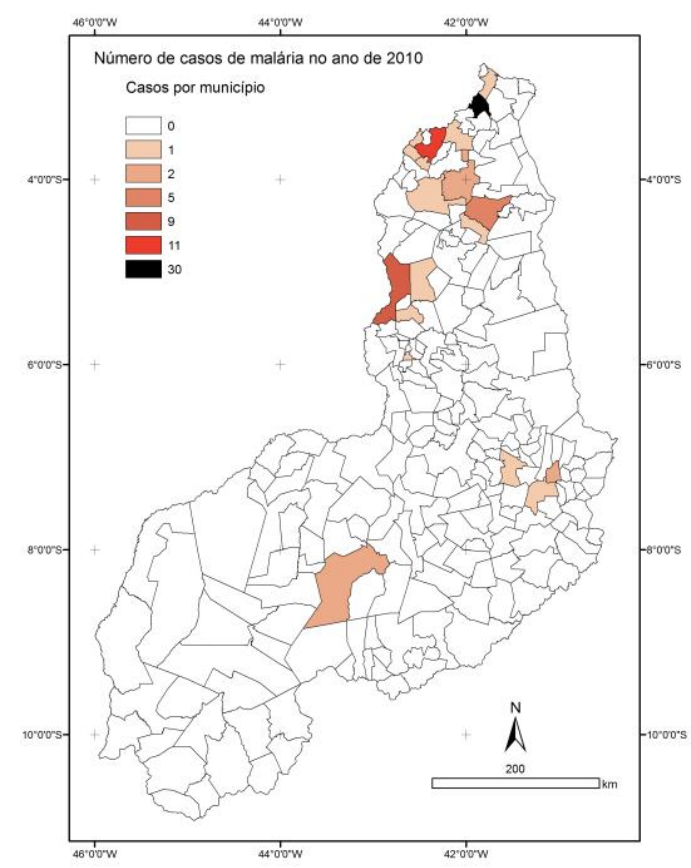

Fonte: SINAN/SESAPI, adaptado por Antonio Carlos dos Santos, 2014.
Figura 5 - Mapa de distribuição espacial dos casos de malária no Piauí no ano de 2011 por município.

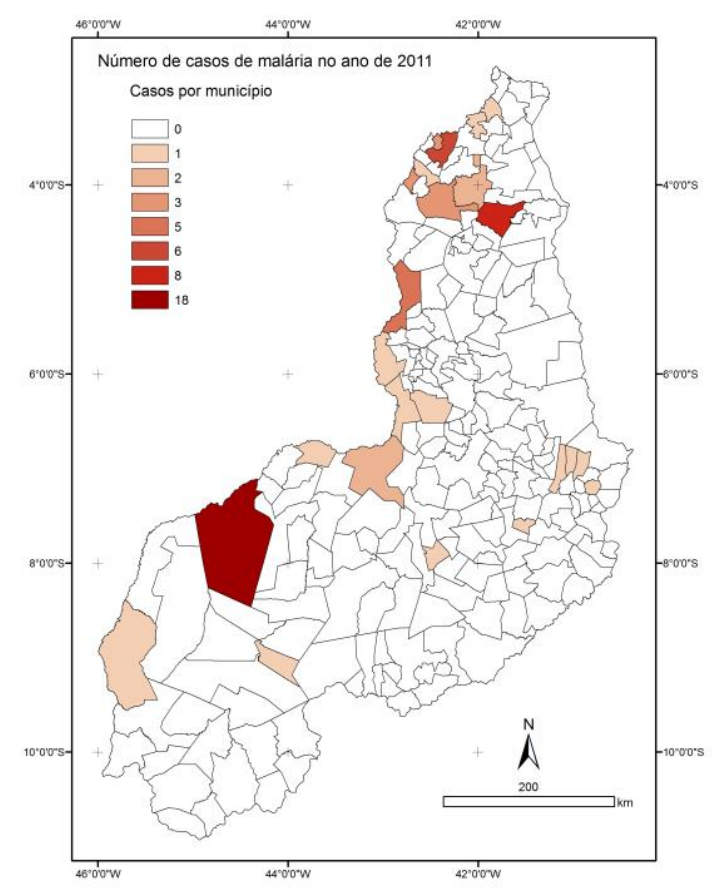

Fonte: SINAN/SESAPI, adaptado por Antonio Carlos dos Santos, 2014.

No ano de 2012 foi notificado um total de 47 casos de malária distribuídos em 20 municípios do estado do Piauí, sendo 13 casos autóctones e 34 casos importados. Teresina notificou o maior número de casos no ano, nove casos, sendo todos importados. Oito municípios notificaram casos de malária autóctone, sendo eles: Miguel Alves com três casos, Brasileira, Matias Olímpio e São Gonçalo do Piauí com registro de dois casos cada um, Boqueirão do Piauí, Campo Largo do Piauí, Luzilândia e Piripiri notificou um caso cada (Figura 6).

Em 2013 (Figura 7) foram notificados 48 casos de malária distribuídos em 15 municípios, sendo 18 autóctones e 30 importados. O município que notificou maior número de casos em 2013 foi Teresina com 13 casos importados. Neste ano cinco municípios notificaram casos de malária autóctone, sendo que Campo Largo do Piauí teve o maior número de casos no total de 12, caracterizando surto. Luzilândia e Porto registraram dois casos autóctones cada um. Pedro II e São José do Divino registraram um caso autóctone cada um. O padrão espacial de distribuição dos casos de malária permanece estável em relação ao ano de 2012. Observa-se também que houve redução no número de municípios que notificaram malária, mas o total de casos foi o mesmo, com aglomeração na porção noroeste do estado. 
Figura 6 - Mapa de distribuição espacial dos casos de malária no Piauí no ano de 2012 por município.

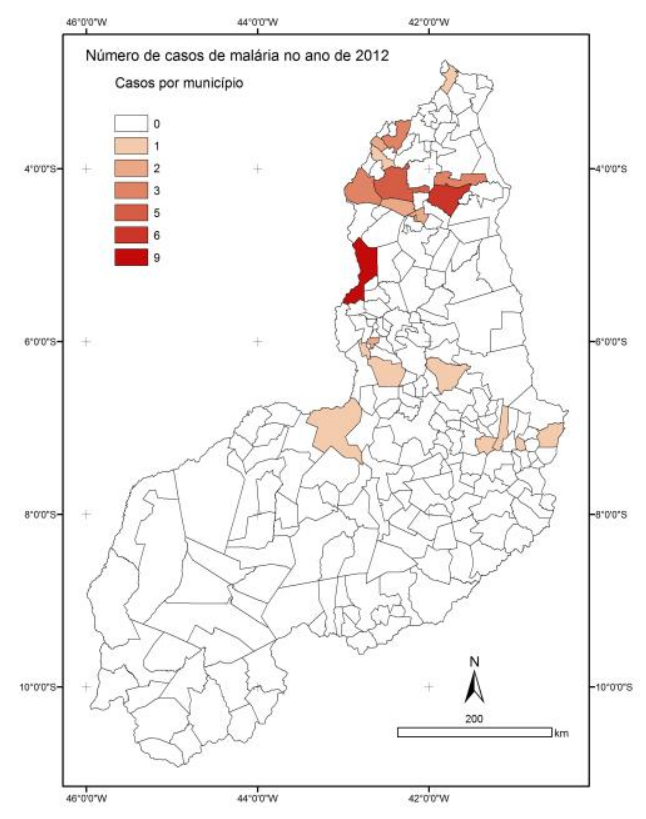

Fonte: SINAN/SESAPI, adaptado por Antonio Carlos dos Santos, 2014.
Figura 7 - Mapa de distribuição espacial dos casos de malária no Piauí no ano de 2013 por município.

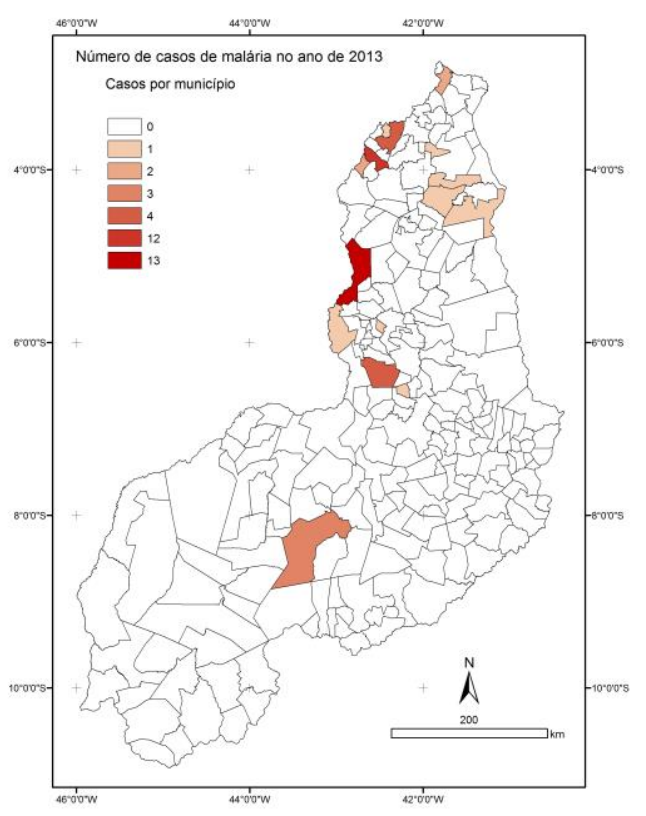

Fonte: SINAN/SESAPI, adaptado por Antonio Carlos dos Santos, 2014.

Segundo Costa et al., (2014), a maioria dos casos de malária diagnosticados e relatados fora da Amazônia são importados da própria Amazônia, da América Central, de outros países da América do Sul, da África e da Ásia, que tem transmissão ativa. No estado do Piauí os casos de malária importados entre 2008 e 2013 foram provenientes dos estados da Amazônia brasileira e países limítrofes dessa região, ou seja, da América do Sul (PIAUÍ, 2009). Os surtos de malária ocorridos nos municípios de Campo Largo do Piauí e Porto em 2013, Buriti dos Lopes em 2010 e Uruçuí em 2011, foram provenientes de estados da região norte do Brasil e de países da América do Sul como Suriname e as Guyanas.

O surto de malária de 2010, com mais de 25 casos em Buriti dos Lopes destacou-se em relação aos demais do período estudado (2008-2013). Segundo SESAPI (PIAUÍ, 2010) no consolidado geral, o sexo masculino foi o mais tingido na época do surto de Buriti dos Lopes - PI, com 61,5\% do total, as faixas etárias com maioria de casos foram a de: 20 a 24 para o sexo masculino, e as de 01 a 04 e 05 a 09 para o sexo feminino. Os principais sintomas descritos por pessoas do município foram: cefaléia, febre alta e intermitente, sudorese abundante, náuseas e vômitos. Com relação à atividade ocupacional registrase que a de lavrador representa $50 \%$ da atividade laboral dos casos maláricos, seguida pela estudantil com $30,8 \%$ dos casos. O surto de 26 casos autóctones em Buriti dos Lopes pode ser considerado como um 
efeito pepita (a variação da elevação em um ano seguido de decréscimo drástico no outro, caracterizando descontinuidade).

\section{CONCLUSÃO}

O mapeamento da espacialização dos casos de malária do ano de 2008 a 2013 no Piauí com uso de Sistema de Informação Geográfica - SIG foi eficiente e prático, pois permitiu uma visão ampla da distribuição dos mesmos e a identificação do padrão espacial.

Através da leitura dos mapas observou-se que a ocorrência de malária é distribuída por todo estado do Piauí considerando os casos como todo, ou seja, importados mais autóctones. Percebeu-se também que houve um significativo aumento da área geográfica com transmissão de malária, partindo do ano de 2008 com incidência em treze municípios e chegando a atingir 24 municípios em 2011.

De acordo com os dados da Tabela 01, das análises das figuras 2 a 7 e levando em consideração os surtos de malária que houve durante a série epidemiológica estudada (2008-2013) pode-se definir que o padrão espacial dos casos de malária no Piauí está concentrado na porção noroeste a norte do estado margeando o rio Parnaíba. Resultado este que comprova a hipótese desse estudo.

Diante da análise dos dados que compôs este artigo conclui-se que a espacialização dos casos de malária no Piauí no período de 2008 a 2013 mostra que 58 de seus municípios estão localizados e aglomerados em áreas vulnerável e receptiva para referida enfermidade o que representa cerca de $26 \%$ do total de seus municípios. Sugere-se à implementação da Vigilância epidemiológica e entomológica no estado, através da SESAPI, com a ferramenta do SIG, objetivando prevenir e monitorar possíveis surtos da doença.

\section{AGRADECIMENTOS}

A Fundação de Amparo à Pesquisa do Estado do Piauí - FAPEPI pelo financiamento da presente pesquisa.

A Secretária Estadual de Saúde do Piauí - SESAPI pela liberação dos dados de malária no SINAN. 


\section{REFERÊNCIAS}

BRASIL. Ministério da Saúde/Fundação Nacional de Saúde. Ações de Controle de Endemias: Malária: Manual para Agentes Comunitários de Saúde e Agentes de Controle de Endemias. Editora MS. Brasília DF, 2002.

CAValcanti, A. P. B. Métodos e Técnicas da Pesquisa Ambiental. UFPI/CCHL/DGH, Teresina PI, 2006.

CHAGAS, F. B.; ARAÚJO, T. M. E.; SANTOS, A. C.; NETO, F. M. C.; SILVA, A. C. L.; LIMA, I. P. Aspectos epidemiológicos e entomológicos de malária autóctone no município de Uruçuí - Piauí - Brasil. In: XLIX CONGRESSO DA SOCIEDADE BRASILEIRA DE MEDICINA TROPICAL, 08/2013, Campo Grande - MS. Anais... Campo Grande - MS: SBMT, 2013. (CD-ROM).

COSTA, A. P.; BRASIL, P.; DI SANTI, S. M.; ARAÚJO, M. P.; SUÁREZ-MUTIS, M. C.; SANTELLI, A. C. S.; FERREIRA, J. O.; OLIVEIRA, R. L.; RIBEIRO, C. T. D. Malária in Brazil: what happens outside the Amazonian endemic region.Revista Memórias do Instituto Oswaldo Cruz, vol.109, n. 5, p.618-633, Rio de Janeiro - RJ, Agosto de 2014.

FILHO, N. de A.; ROUQUAYROL, M. Z. Introdução a Epidemiologia Moderna. Co-edição ABRASCO. Apce Produtos do Conhecimento. 1990. p. $94-101$.

INSTITUTO BRASILEIRO DE GEOGRAFIA E ESTATÍSTICA. Censo demográfico - 2010. Rio de Janeiro: IBGE, 2012.

PIAUÍ - Secretária de Saúde do Estado do Piauí - SESAPI. Relatório de Avaliação Epidemiológicas e Entomológica do Programa Estadual de Controle da Malária - PCM de 1995 a 2008. Teresina, 2009.

PIAUÍ. Secretaria de Saúde de Estado/SESAPI. Relatório técnico do foco de malária autóctone no município de Buriti dos Lopes - PI. Teresina - PI. 2010.

RODRIGUES. J. L. P.Geografia e História do Piauí: Estudos Regionais do Piauí. Editora Halley S/A. 5 ed. Teresina - PI. 2012.

SANTOS, A. C. Estudo Geoambiental da ocorrência de malária no Piauí: Estudo Campo Largo do Piauí e Porto, 2002-2013. Teresina, 2015. Dissertação de Mestrado 114p. UFPI.

SANTOS, A.C.; CHAGAS, M. F. B.; LIMA, I. P.; VAlladARES, G. S.; ARAúJO, T. M. E. Distribuição espacial dos anofelinos (Diptera: Culicidae) no município de Uruçuí (Piauí). In: XIII Reunião Nacional de Pesquisa em Malária, 11/2013, Manaus - AM. Anais XIII Reunião Nacional de Pesquisa em Malária, 11/2013, Manaus - AM: FMT, 2013. (PEN DRIVE).

SANTOS, A. C.; CHAGAS, M. F. B. ; LIMA, I. P. ; VAlLADARES, G. S. ; ARAUJO, T. M. E. . Análise Geoespacial da ocorrência de casos de malária em Uruçuí - PI de janeiro a junho de 2011. In: XI Congresso Nacional de Meio Ambiente de Poços de Caldas - MG, 2014, Poços de Caldas - MG. Anais do XI Congresso Nacional de Meio Ambiente de Poços de Caldas - MG. V. 06, N.1, 2014. Poços de Caldas - MG: IFSULMINAS, 2014. ISSN [2317 - 9686]. Página de 01 a 08. 
Em uma página separada, sendo está a última, deve-se selecionar o eixo-temático a ser submetido o texto e o tipo de apresentação que se deseja realizar. Está página não deve ser contabilizado como número de páginas do texto.

\section{Eixo Temático:}

ET1: Dinâmica dos sistemas ambientais e a saúde

ET2: Acesso e acessibilidades ao sistema de saúde

X ET3: Dados e análises: os desafios do uso das geotecnologias em saúde

ET4: Abordagens e métodos em geografia da saúde

ET5: Alternativas e alternatividades em práticas de saúde coletiva

\section{Tipo de Comunicação:}

1: Comunicação oral

$\mathrm{x}$ 2: Pôster

Para outras informações sobre normatização do texto completo, os autores podem dirigir e-mail a geosaude2015@unb.br 